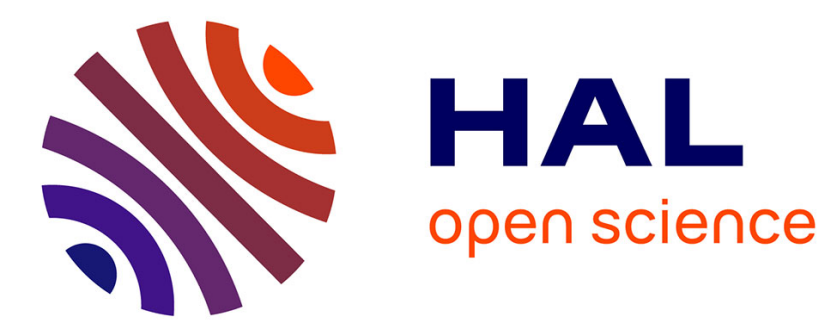

\title{
Transverse instability of gravity-capillary solitary waves on deep water in the presence of constant vorticity
}

\author{
M. Abid, C. Kharif, H.-C. Hsu, Y.-Y. Chen
}

\section{To cite this version:}

M. Abid, C. Kharif, H.-C. Hsu, Y.-Y. Chen. Transverse instability of gravity-capillary solitary waves on deep water in the presence of constant vorticity. Journal of Fluid Mechanics, 2019, 871, pp.10281043. 10.1017/jfm.2019.350 . hal-02070298v2

\section{HAL Id: hal-02070298 \\ https://hal.science/hal-02070298v2}

Submitted on 28 Apr 2020

HAL is a multi-disciplinary open access archive for the deposit and dissemination of scientific research documents, whether they are published or not. The documents may come from teaching and research institutions in France or abroad, or from public or private research centers.
L'archive ouverte pluridisciplinaire HAL, est destinée au dépôt et à la diffusion de documents scientifiques de niveau recherche, publiés ou non, émanant des établissements d'enseignement et de recherche français ou étrangers, des laboratoires publics ou privés. 


\title{
Transverse instability of gravity-capillary solitary waves on deep water in the presence of constant vorticity
}

\author{
M. Abid ${ }^{1}$, C. Kharif ${ }^{1} \dagger$, H.-C. Hsu ${ }^{2}$, and Y.-Y. Chen ${ }^{2}$ \\ ${ }^{1}$ Aix Marseille Université, CNRS, Centrale Marseille, IRPHE UMR 7342, 13384, Marseille, \\ France \\ ${ }^{2}$ Department of Marine Environment and Engineering, National Sun Yat-sen University, \\ Kaohsiung, 801 Taiwan.
}

(Received $\mathrm{xx}$; revised $\mathrm{xx}$; accepted $\mathrm{xx}$ )

The bifurcation of two-dimensional gravity-capillary waves into solitary waves when the phase velocity and group velocity are nearly equal is investigated in the presence of constant vorticity. We found that gravity-capillary solitary waves with decaying oscillatory tails exist in deep water in the presence of vorticity. Furthermore we found that the presence of vorticity influences strongly (i) the solitary wave properties and (ii) the growth rate of unstable transverse perturbations. The growth rate and bandwidth instability are given numerically and analytically as a function of the vorticity.

Keywords: NLS equation, gravity-capillary solitary waves, transverse instability, vorticity

\section{Introduction}

We consider the bifurcation of gravity-capillary waves propagating at the free surface of a vertically sheared current of constant vorticity when the phase and group velocities are nearly equal. In the absence of vorticity, the existence of steadily propagating solitary gravity-capillary waves on deep water was proved numerically by Longuet-Higgins (1989) . Iooss \& Kirchgässner (1990) investigated the problem mathematically and found it was a 1:1 resonance phenomenon. Later on, Vanden-Broeck \& Dias (1992) established the link between the numerical calculation of Longuet-Higgins (1989) and the rigorous analysis of Iooss \& Kirchgässner (1990). Dias et al. (1996) investigated numerically the behavior of these waves in arbitrary depth away from their bifurcation point. Longuet-Higgins (1993) and Akylas (1993) demonstrated that gravity-capillary solitary waves occur when the phase velocity of the carrier wave is equal to the group velocity of the envelopesoliton of the NLS equation. Note that the linear dispersion relation of gravity-capillary waves has a minimum in phase velocity which is equal to the group velocity. For values of the phase velocity less than this extremum the NLS equation admits envelope-soliton solutions such that the wave crests are stationary in the frame of reference of the wave envelope. Later on, steady three-dimensional gravity-capillary solitary waves have been investigated by several authors, among them Parau et al. (2005), Kim \& Akylas (2005) and Milewski (2005).

Gravity-capillary solitary waves have been observed experimentally in wind waves generated in a wind wave facility by Zhang \& Cox (1994). During laboratory experiments (Zhang (1995)) and field experiments (Zhang (1999)) on wind waves on deep water,

$\dagger$ Email address for correspondence: kharif@irphe.univ-mrs.fr. 
gravity-capillary solitary waves were observed and found to closely match numerical profiles obtained by Longuet-Higgins (1989) and Vanden-Broeck \& Dias (1992). LonguetHiggins \& Zhang (1997) generated experimentally gravity-capillary solitary waves on deep water by the resonant excitation of surface waves at a velocity below the minimum of gravity-capillary waves. The mechanism of generation is due to a jet of air impinging on the surface of steady current. Forced three-dimensional gravity-capillary solitary waves on deep water (called lumps) have been investigated experimentally by several authors (Dorio et al. (2011), Masnadi \& Duncan (2017a), Masnadi \& Duncan (2017b) and Park \& Cho (2016)).

To the best of our knowledge, there is no study regarding the effect of constant vorticity on the transverse instability of two-dimensional envelope solitons. On the contrary, many investigations on the transverse instability have been carried out in the absence of vorticity. Zakharov \& Rubenchik (1974) and Saffman \& Yuen (1978) demonstrated that plane gravity envelope solitons are unstable to long wave transverse perturbations. Later on, Ablowitz \& Segur (1979) extended their analyses to show equivalent results in the case of finite depth. Deconinck et al. (2006) obtained a variety of transverse instabilities of gravity envelope solitons in infinite depth. Within the framework of the fully nonlinear potential flow equations Kim \& Akylas (2007) found that plane gravity-capillary solitary waves of depression are unstable to transverse perturbations. Kim (2012) verified the long wave transverse instability within the framework of the weakly nonlinear third-order truncation model. The presence of transverse instability suggests the existence of solitary lumps which are the three-dimensional counterpart of plane gravity-capillary solitary waves. Our purpose is not to consider three-dimensional localized solitary gravity-capillary waves. Akers \& Milewski (2009), Akers \& Milewski (2010), Wang \& Milewski (2012) and Milewski \& Wang (2014) used several model equations to investigate numerically the stability of two-dimensional and three-dimensional gravity-capillary solitary waves in deep water. Regarding two-dimensional waves they found that both gravity-capillary solitary waves of elevation and depression are unstable to transverse perturbations.

The purpose of the present paper is twofold: (i) to revisit the problem of two-dimensional gravity-capillary waves of solitary type on deep water when the effect of an underlying vertically sheared current is taken into account and (ii) to investigate the stability of one-dimensional gravity-capillary solitons, solution of the NLS equation, to transverse perturbation in the presence of constant vorticity.

Section 2 is devoted to the computation of the bifurcated gravity-capillary solitary wave on deep water in the presence of constant vorticity. In section 3 the stability of its envelope to infinitesimal transverse perturbations is investigated.

\section{Gravity-capillary solitary waves with constant vorticity}

We consider envelope soliton bifurcation from a uniform gravity-capillary wave train in the presence of vorticity. Our study is confined to two-dimensional water waves propagating in infinite depth. The fluid is considered inviscid and incompressible. The waves move along the $x$-axis and the $z$-axis is oriented upwards opposite to the gravity $\mathbf{g}=(0,-g)$. The origin $z=0$ is the undisturbed free surface. The waves are travelling at the surface of a vertically sheared current of constant vorticity. In the fixed frame the underlying current is given by $U(z)=U_{0}+\Omega z$ where $\Omega$ is the shear intensity and $U_{0}$ is the current velocity at the surface. Note that the vorticity is $-\Omega$. We choose a reference frame moving with the horizontal velocity $U_{0}$. Consequently, in this frame of reference the current at the surface vanishes and the underlying current is given by $U(z)=\Omega z$. 
Very recently, Hsu et al. (2018) derived a nonlinear Schrödinger equation for the envelope of two-dimensional gravity-capillary waves propagating on arbitrary depth at the free surface of a vertically sheared current of constant vorticity $-\Omega$. Herein, we consider the case of infinite depth. This NLS equation for the complex envelope of the elevation is written as follows

$$
i a_{\tau}+\alpha a_{\xi \xi}=\gamma^{*}|a|^{2} a,
$$

where $a$ is the complex envelope of the free surface elevation which depends on slow variables $\xi=\varepsilon\left(x-c_{g} t\right)$ and $\tau=\varepsilon^{2} t$. The group velocity of the carrier wave of wavenumber, $k$, and frequency, $\omega$, is $c_{g}$. We consider weakly nonlinear waves and consequently we set $\varepsilon \ll 1$.

The dispersion coefficient $\alpha$ reads

$$
\alpha=-\frac{\omega}{k^{2}(2+\Omega / \omega)}\left(\rho^{2}-\frac{\kappa}{1+\kappa} \alpha_{1}\right),
$$

with

$$
\alpha_{1}=(1+\Omega / \omega)(1+2 \rho)+2\left(\rho-2 \frac{\kappa}{1+\kappa}(1+\Omega / \omega)\right)
$$

where

$$
\rho=\frac{1+\Omega / \omega}{2+\Omega / \omega}\left(1+\frac{2 \kappa}{1+\kappa}\right) .
$$

The inverse of the Bond number is

$$
\kappa=\frac{k^{2} T}{\rho_{w} g},
$$

where $T$ is the surface tension coefficient and $\rho_{w}$ the water density.

The nonlinear coefficient $\gamma^{*}$ is

$$
\gamma^{*}=\frac{g^{2}}{4 \omega^{2}}\left(\frac{1+\kappa}{1+\Omega / \omega}\right)^{2} \gamma,
$$

where

$$
\begin{aligned}
\gamma= & \frac{k^{4}}{2 \omega(1+\Omega / \omega)(2+\Omega / \omega)}\left[-\frac{3 \kappa}{1+\kappa}(1+\Omega / \omega)^{2}\right. \\
& +(1+\Omega / \omega)(8+6 \Omega / \omega)+\frac{1+\Omega / \omega}{1-\kappa(2+3 \Omega / \omega)} \gamma_{1} \\
& \left.+2 \frac{(1+\Omega / \omega)(2+\Omega / \omega)^{2}}{\rho \Omega / \omega-(1+\Omega / \omega) /(1+\kappa)}(\Omega / \omega)^{2}\right]
\end{aligned}
$$

and

$$
\gamma_{1}=12 \kappa+10(1+4 \kappa) \Omega / \omega+6(3+7 \kappa)(\Omega / \omega)^{2}+2(4+7 \kappa)(\Omega / \omega)^{3}+(1+\kappa)(\Omega / \omega)^{4} .
$$

Equation (2.1) admits the following solution

$$
a= \pm a_{0} \operatorname{sech}\left\{a_{0}\left(\frac{-\gamma^{*}}{2 \alpha}\right)^{1 / 2} \xi\right\} \exp \left(-\frac{i}{2} \gamma^{*} a_{0}^{2} \tau\right)
$$

and the elevation of a weakly nonlinear wave train of envelope $a(\xi, \tau)$ writes

$$
\zeta= \pm \frac{1}{2} \varepsilon a_{0} \operatorname{sech}\left\{a_{0}\left(\frac{-\gamma^{*}}{2 \alpha}\right)^{1 / 2} \xi\right\} \exp \left[i k x-i\left(\omega+\frac{1}{2} \gamma^{*} \varepsilon^{2} a_{0}^{2}\right) t\right]+c . c .,
$$


where $k$ and $\omega$ are the carrier wavenumber and carrier frequency, $\varepsilon \ll 1$ and c.c. denotes the complex conjugation. The wave height at the origin is $\varepsilon a_{0}$.

The crests of the wave group travel at phase velocity

$$
c=\frac{\omega}{k}+\frac{1}{2} \gamma^{*} \varepsilon^{2} a_{0}^{2} / k,
$$

whereas the envelope travels at group velocity $c_{g}$.

The condition for the envelope-soliton to be a solitary wave is that the group velocity is equal to the phase velocity $c_{g}=c$

$$
c_{g}=\frac{\omega}{k}+\frac{1}{2} \gamma^{*} \varepsilon^{2} a_{0}^{2} / k
$$

Whatever $\Omega$, the nonlinear coefficient of the NLS equation is negative (see figure 1), therefore the solitary wave travels at a constant velocity which is a little less than the minimum phase velocity of linear gravity-capillary waves.

Following Longuet-Higgins (1993), we set $g=1, T=1$ and $\rho_{w}=1$ which is equivalent to use $\left(T / \rho_{w} g\right)^{1 / 2}$ and $\left(T / \rho_{w} g^{3}\right)^{1 / 4}$ as unit of length and unit of time, respectively. For the sake of clarity the same symbols are used to define the dimensionless frequency, vorticity and wavenumber. Then, the nonlinear coefficient $\gamma^{*}$ becomes in dimensionless form

$$
\gamma^{*}(k)=\left(\frac{1+k^{2}}{\omega+\Omega}\right)^{2} \gamma / 4
$$

where

$$
\begin{aligned}
\gamma= & \frac{k^{4}}{2 \omega(1+\Omega / \omega)(2+\Omega / \omega)}\left[-\frac{3 k^{2}}{1+k^{2}}(1+\Omega / \omega)^{2}\right. \\
& +(1+\Omega / \omega)(8+6 \Omega / \omega)+\frac{1+\Omega / \omega}{1-k^{2}(2+3 \Omega / \omega)} \gamma_{1} \\
& \left.+2 \frac{(1+\Omega / \omega)(2+\Omega / \omega)^{2}}{\rho \Omega / \omega-(1+\Omega / \omega) /\left(1+k^{2}\right)}(\Omega / \omega)^{2}\right]
\end{aligned}
$$

with

$\gamma_{1}=12 k^{2}+10\left(1+4 k^{2}\right) \Omega / \omega+6\left(3+7 k^{2}\right)(\Omega / \omega)^{2}+2\left(4+7 k^{2}\right)(\Omega / \omega)^{3}+\left(1+k^{2}\right)(\Omega / \omega)^{4}$, and

$$
\rho=\frac{1+\Omega / \omega}{2+\Omega / \omega}\left(1+\frac{2 k^{2}}{1+k^{2}}\right) .
$$

The dimensionless linear dispersion relation is

$$
\omega^{2}+\Omega \omega-k\left(1+k^{2}\right)=0 .
$$

The dimensionless linear phase velocity and group velocity are equal at the linear phase velocity minimum which occurs when $k$ is solution of the following equation

$$
k^{2}+\Omega \sqrt{2} k^{1 / 2}-1=0 .
$$

For the case $\Omega=0$ which was considered by Longuet-Higgins (1993) we find $k=1$, $\omega=\sqrt{2}$ and $\gamma^{*}(k)=-11 \sqrt{2} / 32$. Then, the velocity of the solitary wave is

$$
c_{g 0}=\sqrt{2}\left(1-\frac{11}{64} \varepsilon^{2} a_{0}^{2}\right),
$$

which is the equation (3.19) of Longuet-Higgins (1993). 
Equation $(2.3)$ admits the following aproximate solution

$$
k=1-\frac{\Omega}{\sqrt{2}}+\mathcal{O}\left(\Omega^{2}\right) .
$$

Then

$$
\omega=\sqrt{2}-\frac{3}{2} \Omega+\mathcal{O}\left(\Omega^{2}\right)
$$

Negative vorticity $(\Omega>0)$ reduces $k$ and $\omega$ whereas positive vorticity $(\Omega<0)$ increases $k$ and $\omega$.

An approximate solution of equation 2.2$)$ is

$$
c_{g}=\sqrt{2}-\frac{\Omega}{2}-\left(\frac{11 \sqrt{2}}{64}+\frac{31}{64} \Omega\right) \varepsilon^{2} a_{0}^{2}+\mathcal{O}\left(\Omega^{2}, \varepsilon^{3} a_{0}^{3}\right),
$$

which can be rewritten as follows

$$
c_{g}=c_{g 0}-\frac{\Omega}{2}\left(1+\frac{31}{32} \varepsilon^{2} a_{0}^{2}\right)+\mathcal{O}\left(\Omega^{2}, \varepsilon^{3} a_{0}^{3}\right)
$$

Solitary wave velocity is increased when the vorticity is positive $(\Omega<0)$ and reduced when the vorticity is negative $(\Omega>0)$.

To extend the work of Akylas (1993), we investigate the bifurcation of a train of periodic gravity-capillary waves into a solitary wave with decaying oscillatory tail in the presence of constant vorticity. Now, we use $T /\left(\rho_{w} c^{2}\right)$ and $T /\left(\rho_{w} c^{3}\right)$ as unit length and unit time where $c$ is the phase velocity of the envelope-soliton, which means that $c$ is the reference phase velocity scale. Note that these time and length units were used by Vanden-Broeck \& Dias (1992) and Akylas (1993). Consequently, with these new scaled variables the dimensionless form of the NLS equation 2.1 becomes

$$
i \tilde{a}_{\tilde{\tau}}+\tilde{\alpha} \tilde{a}_{\tilde{\xi} \tilde{\xi}}=\beta^{2} \tilde{\gamma}^{*}|\tilde{a}|^{2} \tilde{a}
$$

where $\beta=g T /\left(\rho_{w} c^{4}\right), \tilde{\tau}=\rho_{w} c^{3} \tau / T, \tilde{\xi}=\rho_{w} c^{2} \xi / T, \tilde{a}=\rho_{w} c^{2} a / T, \tilde{\alpha}=\rho_{w} c \alpha / T$ and $\tilde{\gamma}^{*}=T c \gamma^{*} /\left(\rho_{w} g^{2}\right)$.

Using $\tilde{\omega}=T \omega /\left(\rho_{w} c^{3}\right), \tilde{k}=T k /\left(\rho_{w} c^{2}\right), \tilde{\Omega}=T \Omega /\left(\rho_{w} c^{3}\right)$ and $\kappa=\tilde{k}^{2} / \beta$ the coefficient $\tilde{\gamma}^{*}$ is

$$
\begin{array}{r}
\tilde{\gamma}^{*}=\frac{\tilde{\omega} \tilde{k}^{4}\left(1+\tilde{k}^{2} / \beta\right)^{2}}{8(\tilde{\omega}+\tilde{\Omega})^{3}(2 \tilde{\omega}+\tilde{\Omega})}\left\{-\frac{3 \tilde{k}^{2}(\tilde{\omega}+\tilde{\Omega})^{2}}{\tilde{\omega}^{2}\left(\beta+\tilde{k}^{2}\right)}+\frac{(\tilde{\omega}+\tilde{\Omega})(8 \tilde{\omega}+6 \tilde{\Omega})}{\tilde{\omega}^{2}}+\frac{(\tilde{\omega}+\tilde{\Omega})}{\beta \tilde{\omega}-\tilde{k}^{2}(2 \tilde{\omega}+3 \tilde{\Omega})}\left(\beta \tilde{\gamma_{1}}\right)\right. \\
\left.+\frac{2(\tilde{\omega}+\tilde{\Omega})(2 \tilde{\omega}+\tilde{\Omega})^{2} \tilde{\Omega}^{2}}{\tilde{\omega}^{4}\left(\tilde{\rho} \tilde{\Omega}-\beta(\tilde{\omega}+\tilde{\Omega}) /\left(\beta+\tilde{k}^{2}\right)\right)}\right\},
\end{array}
$$

where

$$
\beta \tilde{\gamma_{1}}=\beta\left(10 \Delta+18 \Delta^{2}+8 \Delta^{3}+\Delta^{4}\right)+\tilde{k}^{2}\left(12+40 \Delta+42 \Delta^{2}+14 \Delta^{3}+\Delta^{4}\right), \quad \Delta=\frac{\tilde{\Omega}}{\tilde{\omega}},
$$

and

$$
\tilde{\rho}=\frac{(\tilde{\omega}+\tilde{\Omega})(1+2 \kappa /(1+\kappa))}{2 \tilde{\omega}+\tilde{\Omega}} .
$$

Let $\tilde{\mu}=\beta^{2} \tilde{\gamma}^{*}$. For $\tilde{\Omega}=0$, we have checked that $-\tilde{\mu}$ is equal to the expression derived by Akylas (1993). In figure 1 is plotted the nonlinear coefficient $\tilde{\mu}$ as a function of $\Omega$. An enlargement is inserted in the figure in the vicinity of $\Omega \approx-0.2$ to show that this 


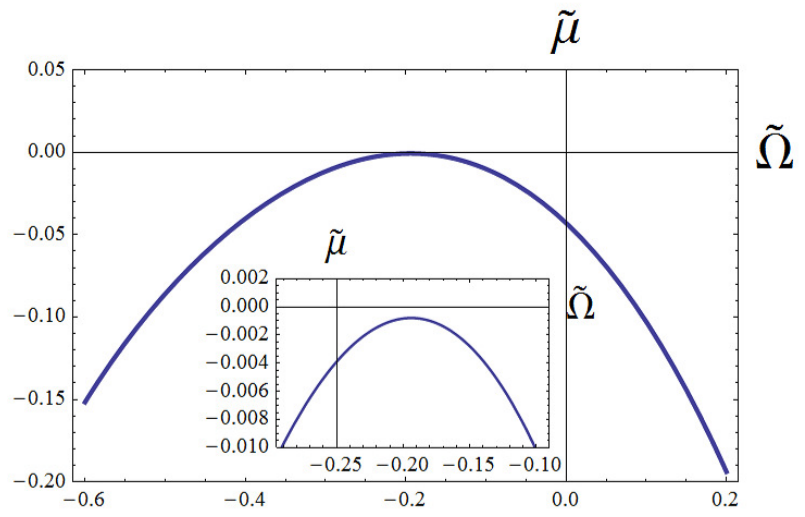

Figure 1. Dimensionless nonlinear coefficient, $\tilde{\mu}$, of the NLS equation as a function of the vorticity.

coefficient is always negative.

The dimensionless form of the dispersive coefficient is

$$
\tilde{\alpha}=-\frac{\tilde{\omega}^{2}}{\tilde{k}^{2}(2 \tilde{\omega}+\tilde{\Omega})}\left(\tilde{\rho}^{2}-\frac{\tilde{k}^{2}}{\beta+\tilde{k}^{2}} \tilde{\alpha}_{1}\right),
$$

where

$$
\tilde{\rho}=\frac{\tilde{\omega}+\tilde{\Omega}}{2 \tilde{\omega}+\tilde{\Omega}}\left(1+\frac{2 \tilde{k}^{2}}{\beta+\tilde{k}^{2}}\right)
$$

and

$$
\tilde{\alpha_{1}}=\frac{(\tilde{\omega}+\tilde{\Omega})(1+2 \tilde{\rho})}{\tilde{\omega}}+2\left(\tilde{\rho}-\frac{2 \tilde{k}^{2}(\tilde{\omega}+\tilde{\Omega})}{\tilde{\omega}\left(\beta+\tilde{k}^{2}\right)}\right)
$$

The dimensionless NLS equation 2.6 becomes, dropping the tildes

$$
i a_{\tau}+\alpha a_{\xi \xi}=\mu|a|^{2} a,
$$

and the free surface elevation writes

$$
\zeta= \pm \frac{1}{2} \varepsilon a_{0} \operatorname{sech}\left\{a_{0}\left(\frac{-\mu}{2 \alpha}\right)^{1 / 2} \xi\right\} \exp \left[i k x-i\left(\omega+\frac{1}{2} \mu \varepsilon^{2} a_{0}^{2}\right) t\right]+c . c .
$$

The condition for the envelope-soliton to be a solitary wave is, due to the chosen normalisation, $c_{g}=c=1$

$$
1=\frac{\omega}{k}+\frac{1}{2} \mu \varepsilon^{2} a_{0}^{2} / k
$$

Hence,

$$
k=\omega(k, \Omega, \beta)+\frac{1}{2} \mu \varepsilon^{2} a_{0}^{2} .
$$

For infinite depth, the dimensionless linear gravity-capillary dispersion relation in the presence of a shear of intensity $\Omega$ is

$$
\omega^{2}+\Omega \omega-\beta k-k^{3}=0,
$$


which has a minimum in phase velocity at $k=k_{0}$. At this minimum the phase velocity is equal to the group velocity

$$
k_{0}=\omega\left(k_{0}, \Omega, \beta_{0}\right) \quad \text { and } \quad \frac{\partial \omega}{\partial k}\left(k_{0}, \Omega, \beta_{0}\right)=1 .
$$

Solving these two equations gives

$$
k_{0}=\frac{1}{2}
$$

and

$$
\beta_{0}=\frac{1}{4}+\Omega \text {. }
$$

For $\Omega=0$ the values of Akylas (1993) are rediscovered. Note that the shear has no effect on the dimensionless value of $k_{0}$. Nevertheless, with the scaled variables used by Longuet-Higgins (1993) equation 2.10 reduces to equation 2.4 at $\mathcal{O}(\Omega)$.

Herein, $\omega>0$ and consequently $\omega_{0}=1 / 2$. From the dispersion relation $(2.9)$, it can be demonstrated that we always have $-\Omega<\omega$ or $-\omega<\Omega$. In our case the carrier wave frequency is close to $\omega_{0}$ and as a result the considered values of $\Omega$ are larger than a value close to $-1 / 2$.

The wavenumber $k$ and $\beta$ are expanded about $\left(k_{0}, \beta_{0}\right)$ as follows

$$
\begin{aligned}
& k=k_{0}+k_{1} \varepsilon^{2}+\mathcal{O}\left(\varepsilon^{3}\right), \\
& \beta=\beta_{0}+\beta_{1} \varepsilon^{2}+\mathcal{O}\left(\varepsilon^{3}\right) .
\end{aligned}
$$

Expanding $\omega(k, \Omega, \beta)$ about $\left(k_{0}, \beta_{0}\right)$ and using equation 2.8 with $\partial \omega / \partial k\left(k_{0}, \Omega, \beta_{0}\right)=1$ gives

and

$$
\beta_{1}=-\frac{1}{2} \frac{\mu\left(k_{0}, \Omega, \beta_{0}\right)}{\partial \omega / \partial \beta\left(k_{0}, \Omega, \beta_{0}\right)} a_{0}^{2},
$$

$$
k_{1}=-\frac{\frac{\partial^{2} \omega}{\partial \beta \partial k}\left(k_{0}, \Omega, \beta_{0}\right)}{\frac{\partial^{2} \omega}{\partial k^{2}}\left(k_{0}, \Omega, \beta_{0}\right)} \beta_{1} .
$$

Using

we obtain

$$
\frac{\partial^{2} \omega}{\partial k^{2}}\left(k_{0}, \Omega, \beta_{0}\right)=2 \alpha\left(k_{0}, \Omega, \beta_{0}\right)
$$

$$
k_{1}=-\frac{\frac{\partial^{2} \omega}{\partial \beta \partial k}\left(k_{0}, \Omega, \beta_{0}\right)}{2 \alpha\left(k_{0}, \Omega, \beta_{0}\right)} \beta_{1} .
$$

Let $\delta \beta$ be the difference between $\beta$ and the critical value $\beta_{0}$

Knowing that

$$
\begin{gathered}
\delta \beta=\beta_{1} \varepsilon^{2}+\mathcal{O}\left(\varepsilon^{3}\right), \\
\delta \beta=-\frac{1}{2} \frac{\mu\left(k_{0}, \Omega, \beta_{0}\right)}{\partial \omega / \partial \beta\left(k_{0}, \Omega, \beta_{0}\right)} a_{0}^{2} \varepsilon^{2} .
\end{gathered}
$$

$$
\partial \omega / \partial \beta\left(k_{0}, \Omega, \beta_{0}\right)=\frac{1}{2(1+\Omega)},
$$


then

$$
a_{0}^{2} \varepsilon^{2}=-\frac{\delta \beta}{(1+\Omega) \mu\left(k_{0}, \Omega, \beta_{0}\right)} .
$$

From equation 2.11 and figure 1 we can see that the wave height decreases as positive values of $\Omega$ increases. For negative values of $\Omega$ the wave height presents a maximum in the vicinity of $\Omega \approx-0.2$. Finally,

$$
a_{0}^{2} \varepsilon^{2}=\frac{16^{2} \delta \beta}{11+128 \Omega+464 \Omega^{2}+512 \Omega^{3}+192 \Omega^{4}} .
$$

Knowing that

$$
\frac{\partial^{2} \omega}{\partial \beta \partial k}\left(k_{0}, \Omega, \beta_{0}\right)=\frac{\Omega}{(1+\Omega)^{2}},
$$

and

then

$$
\frac{\partial^{2} \omega}{\partial k^{2}}\left(k_{0}, \Omega, \beta_{0}\right)=2 \alpha\left(k_{0}, \Omega, \beta_{0}\right)=\frac{1}{1+\Omega},
$$

$$
k_{1}=-\frac{\Omega}{1+\Omega} \beta_{1}
$$

and

$$
k=k_{0}+k_{1} \varepsilon^{2}=k_{0}-\frac{\Omega}{1+\Omega} \beta_{1} \varepsilon^{2}=k_{0}-\frac{\Omega}{1+\Omega} \delta \beta .
$$

Note that in the absence of vorticity the dimensionless carrier wavenumber is unchanged at $\mathcal{O}\left(\varepsilon^{2}\right)$. Negative vorticity $(\Omega>0)$ reduces $k$ whereas positive vorticity $(\Omega<0)$ increases $k$.

The expression of the solitary wave is

$\zeta(x, t)= \pm 16\left(\frac{\delta \beta}{11+128 \Omega+464 \Omega^{2}+512 \Omega^{3}+192 \Omega^{4}}\right)^{1 / 2} \operatorname{sech}\left\{(\delta \beta)^{1 / 2}(x-t)\right\} \cos \{k(x-t)\}$.

The profile of the solitary wave is known once the bifurcation parameter $\delta \beta$ is fixed. Equations 2.12 and (2.13) allows the determination of the parameter $\varepsilon a_{0}$ and $k$, respectively. Profiles of solitary waves of elevation and depression which bifurcate at $\beta=\beta_{0}$ from a linear uniform periodic wave train of frequency $\omega_{0}$ and wavenumber $k_{0}$, are plotted in figures $2,3,4$ and 5 . For negative vorticity $(\Omega>0)$, an increase of $\Omega$ leads to a decrease of the wave height (see figures 2 and 4 ). For positive vorticity $(\Omega<0)$, an increase of $|\Omega|$ may lead to an increase (see figures $3,5,6$ and 7) of wave height.

\section{Transverse instability of the envelope of gravity-capillary solitary waves with constant vorticity}

The two-dimensional version of the NLS equation in deep water (2.1) is

$$
i a_{\tau}+\alpha a_{\xi \xi}+\lambda a_{\eta \eta}+2 \Gamma|a|^{2} a=0,
$$

where $\eta$ is the transverse coordinate and $\Gamma=-\gamma^{*} / 2$.

The transverse dispersion coefficient $\lambda$ reads

$$
\lambda=\frac{\omega^{\prime}}{2 k}=\frac{g(1+3 \kappa)}{4 k(\omega+\Omega / 2)} .
$$




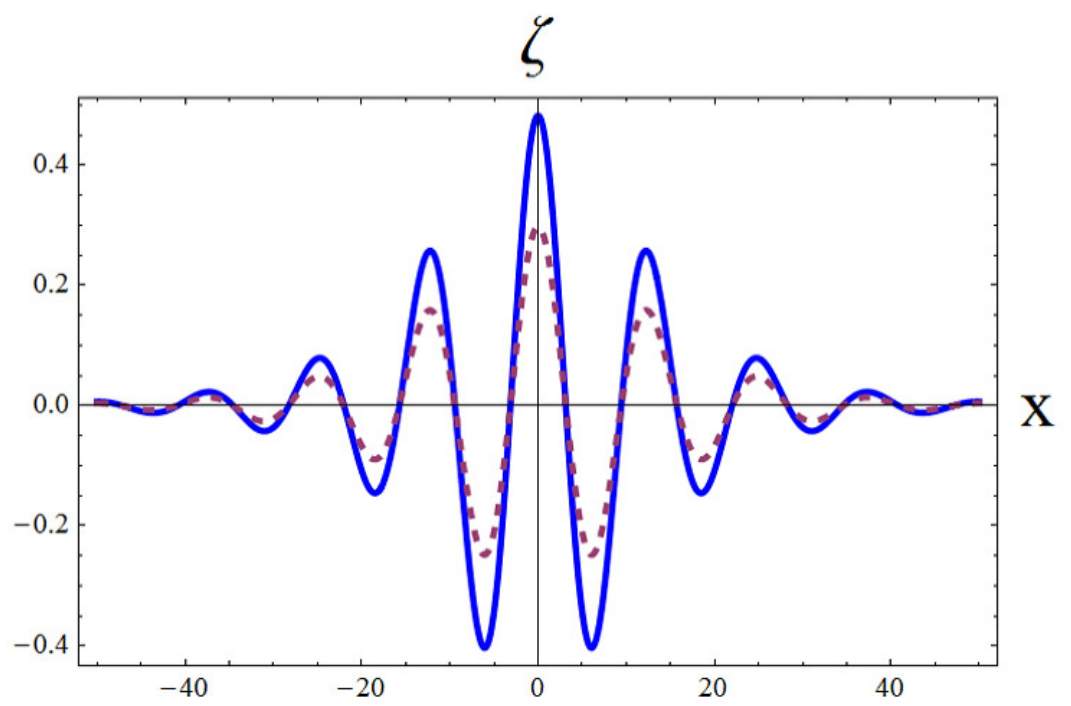

Figure 2. Dimensionless profiles of solitary waves of elevation: Solid line $(\Omega=0)$ and dashed line $(\Omega=0.10)$.

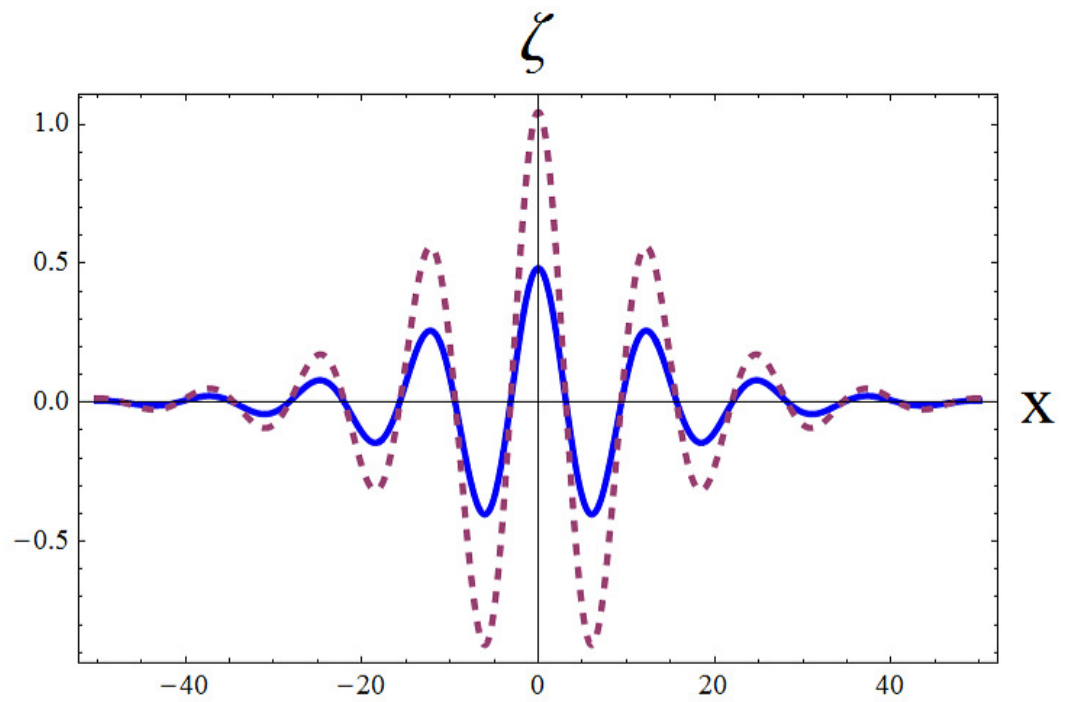

Figure 3. Dimensionless profiles of solitary waves of elevation: Solid line $(\Omega=0)$ and dashed line $(\Omega=-0.1)$.

For $\partial / \partial \eta=0$, equation 3.1 admits the following solution

$$
\bar{a}=A(\xi) \exp \left(i \Gamma a_{0}^{2} \tau\right)
$$

where

$$
A(\xi)=a_{0} \operatorname{sech}\left\{a_{0}\left(\frac{\Gamma}{\alpha}\right)^{1 / 2} \xi\right\}
$$




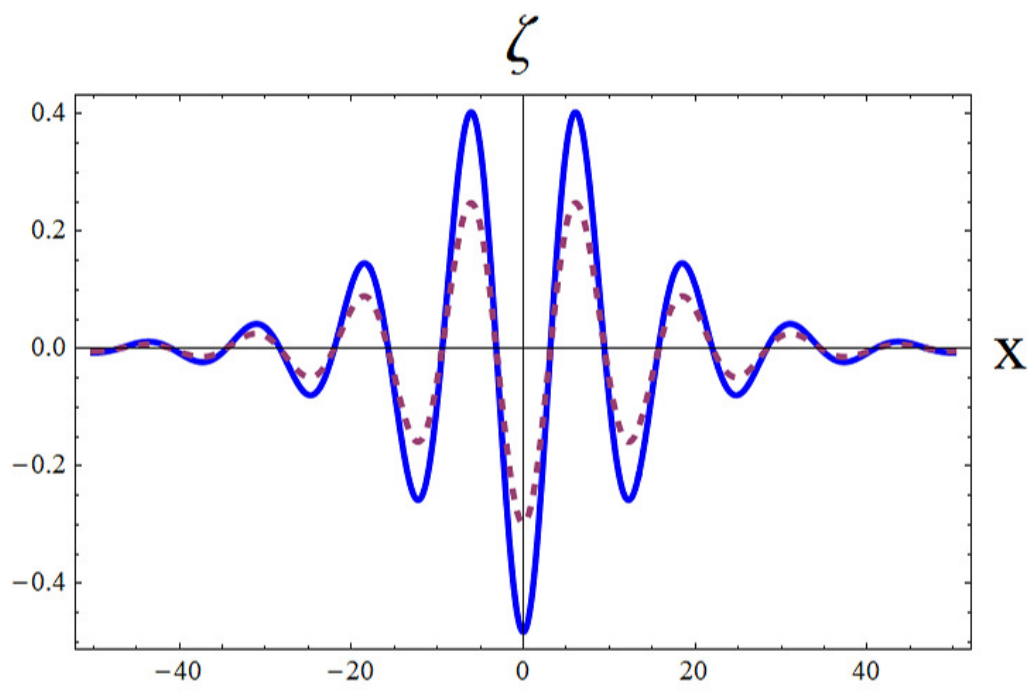

Figure 4. Dimensionless profiles of solitary waves of depression: Solid line $(\Omega=0)$ and dashed line $(\Omega=0.10)$.

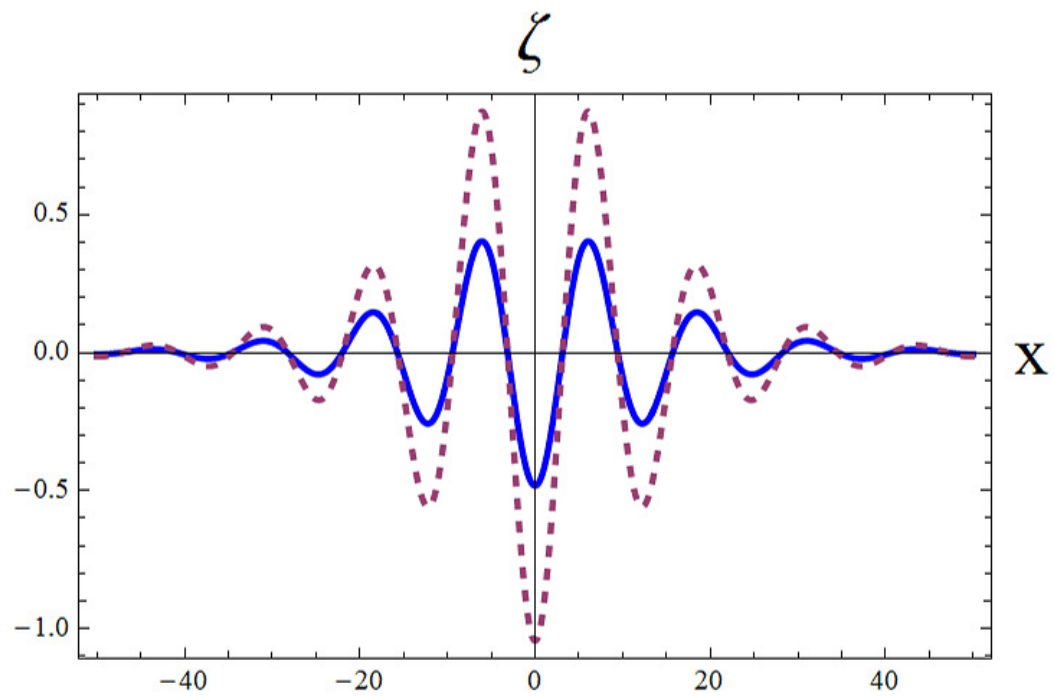

FiguRE 5. Dimensionless profiles of solitary waves of depression: Solid line $(\Omega=0)$ and dashed line $(\Omega=-0.1)$.

Let us perturb with an infinitesimal perturbation the solution 3.2

$$
a=\bar{a}+a^{\prime} \exp \left(i \Gamma a_{0}^{2} \tau\right)
$$

Linearisation of equation $(3.1)$ about $\bar{a}$ gives

$$
i a_{\tau}^{\prime}-\Gamma a_{0}^{2} a^{\prime}+\alpha a_{\xi \xi}^{\prime}+\lambda a_{\eta \eta}^{\prime}=-2 \Gamma A^{2} a^{\prime *}-4 \Gamma A^{2} a^{\prime} .
$$




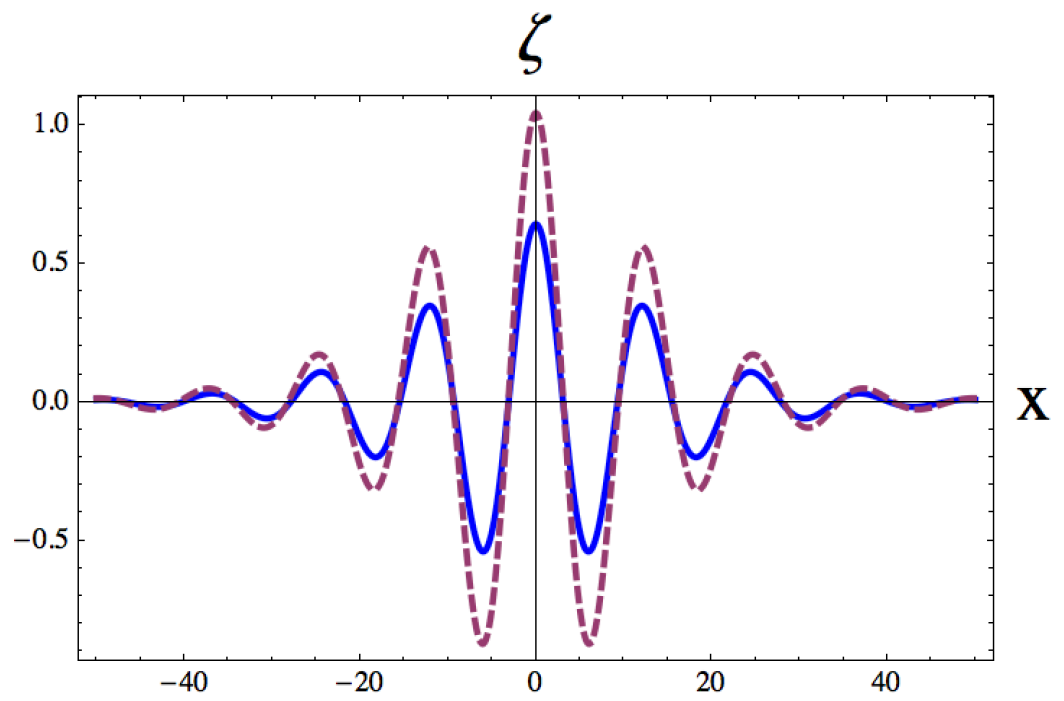

Figure 6. Dimensionless profiles of solitary waves of elevation: Solid line $(\Omega=-0.10)$ and dashed line $(\Omega=-0.4)$.

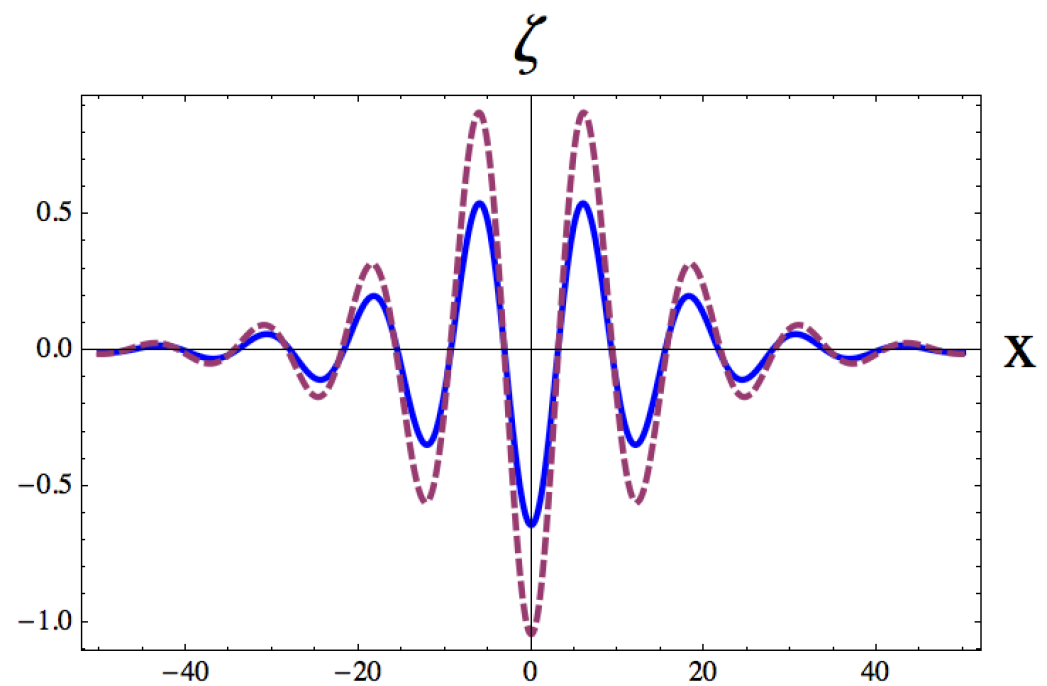

Figure 7. Dimensionless profiles of solitary waves of depression: Solid line $(\Omega=-0.10)$ and dashed line $(\Omega=-0.4)$.

Let us consider $a^{\prime}=u+i v$ (with $u$ and $v$ reals) and substitute this expression into (3.3)

$$
\begin{gathered}
v_{\tau}=\alpha u_{\xi \xi}+\lambda u_{\eta \eta}-\left(-6 \Gamma A^{2}+\Gamma a_{0}^{2}\right) u, \\
u_{\tau}=-\alpha v_{\xi \xi}-\lambda v_{\eta \eta}+\left(-2 \Gamma A^{2}+\Gamma a_{0}^{2}\right) v .
\end{gathered}
$$

The system (3.4)-3.5) of linear partial differential equations with constant coefficients admits solutions of the following forms (normal modes)

$$
u=\hat{u}(\xi) \exp (i q \eta+\sigma \tau),
$$




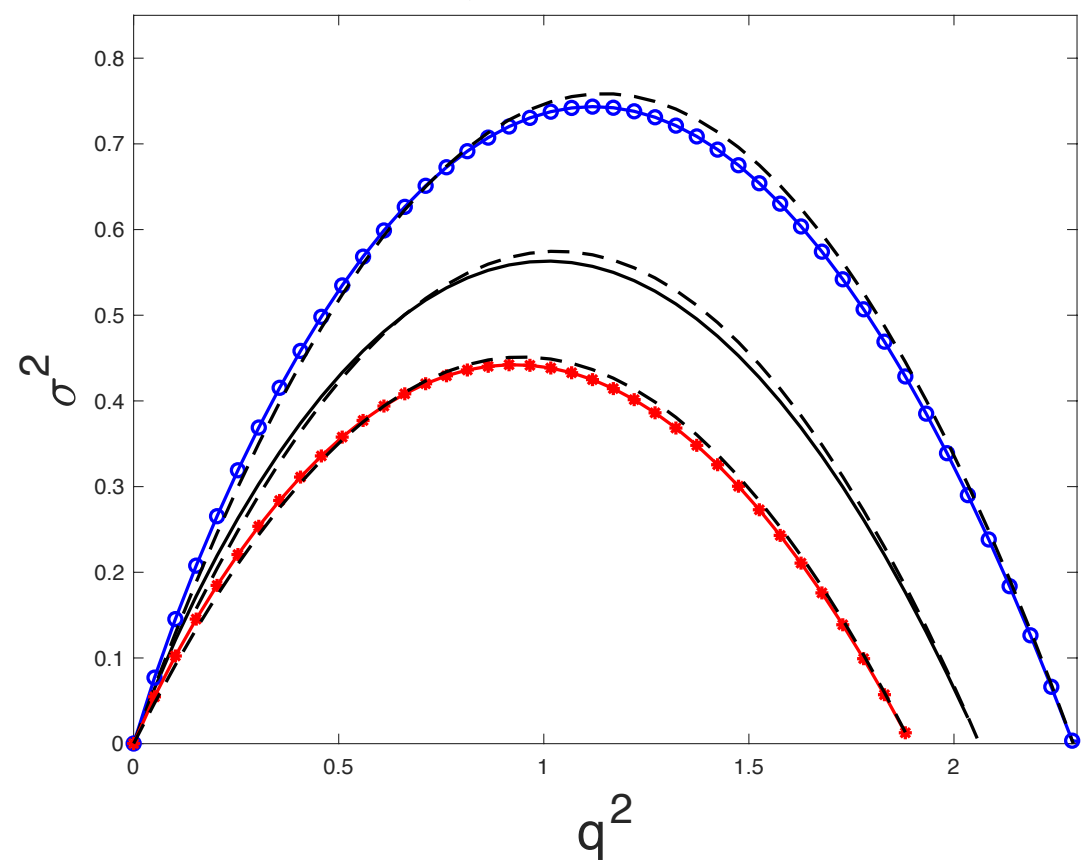

FIGURE 8. Square of the dimensionless growth rate of the transverse unstable mode of the envelope of gravity-capillary solitary waves in deep water as a function of the square of the dimensionless wavenumber for $\Omega=0$ (solid line), $\Omega=0.1$ ( $\circ$ ) and $\Omega=-0.1(\star$ ). The dashed lines correspond to the analytic expression given by equation $3.9 \cdot a_{0}=\sqrt{2}$.

$$
v=\hat{v}(\xi) \exp (i q \eta+\sigma \tau)
$$

Herein we can use the complex forms for $u$ and $v$ because we deal with linear equations. Substitution of $(3.6)$ and $(3.7)$ into 3.5 and 3.4 gives

$$
\begin{gathered}
\sigma \hat{v}=\alpha \frac{d^{2} \hat{u}}{d \xi^{2}}-\lambda q^{2} \hat{u}-\left(-6 \Gamma A^{2}+\Gamma a_{0}^{2}\right) \hat{u}, \\
\sigma \hat{u}=-\alpha \frac{d^{2} \hat{v}}{d \xi^{2}}+\lambda q^{2} \hat{v}+\left(-2 \Gamma A^{2}+\Gamma a_{0}^{2}\right) \hat{v} .
\end{gathered}
$$

The above system can be written as follows

$$
\sigma^{2} \hat{u}=-\left(L_{0}-\lambda q^{2}\right)\left(L_{1}-\lambda q^{2}\right) \hat{u}
$$

with

$$
L_{0}=\alpha \frac{d^{2}}{d \xi^{2}}+2 \Gamma A^{2}-\Gamma a_{0}^{2}
$$

and

$$
L_{1}=\alpha \frac{d^{2}}{d \xi^{2}}+6 \Gamma A^{2}-\Gamma a_{0}^{2},
$$

and the eigenfunction $\hat{u}$ vanishes as $|\xi| \rightarrow \infty$.

The numerical method to solve equation 3.8 is outlined in the Appendix.

We set $g=1, T=1$ and $\rho_{w}=1$. For moderate and small values of the vorticity the coefficients of the NLS equation (3.1) are positive, $\Gamma>0, \alpha>0$ and $\lambda>0$. For comparison we use herein the analytic relation of the instability growth rate given by 
Rypdal \& Rasmussen (1989) which writes in dimensionless form

$$
\sigma^{2}=\frac{64}{\pi^{2}} \lambda \Gamma q^{2}\left(1-\frac{\lambda}{6 \Gamma} q^{2}\right)
$$

The maximum growth rate is

$$
\sigma_{\max }^{2}=\frac{96 \Gamma^{2}}{\pi^{2}}
$$

and

$$
q_{\max }=\sqrt{\frac{3 \Gamma}{\lambda}} .
$$

The gravity-capillary solitary wave is marginally stable for $q=q_{c}$ with

$$
q_{c}^{2}=\frac{6 \Gamma}{\lambda}
$$

For small values of the vorticity the instability growth rate and marginal wavenumber are

and

$$
\sigma^{2}=q^{2}\left(\frac{11}{\pi^{2}}-\frac{16}{3 \pi^{2}} q^{2}\right)+q^{2}\left(\frac{153+32 q^{2}}{6 \sqrt{2} \pi^{2}}\right) \Omega+\mathcal{O}\left(\Omega^{2}\right),
$$

$$
q_{c}=\frac{33}{16}+\frac{87}{33 \sqrt{2}} \Omega+\mathcal{O}\left(\Omega^{2}\right)
$$

In figure 8 is shown the numerical and analytic instability growth rates for several values of the vorticity. The solid lines correspond to numerical solutions of equation (3.8). Negative vorticity amplifies the rate of growth whereas positive vorticity reduces its value. Surface elevation of the envelope of gravity-capillary solitary waves perturbed by their most unstable modes corresponding to $\Omega=0, q=1.01$ and $\Omega=0.10, q=1.08$ are shown in figure 9. These waves which are localized in the direction of propagation and periodic in the transverse direction have been investigated by Milewski \& Wang (2014). This new solution found by the latter authors was called transversally periodic plane solitary wave.

\section{Conclusion}

Within the framework of the $1 D-$ and $2 D-$ NLS equations, the effect of constant vorticity on the properties and stability of gravity-capillary solitary waves in deep water has been investigated. These two-dimensional solitary waves of elevation and depression types bifurcate from linear uniform wavetrains at the phase speed minimum where the phase velocity equals the group velocity. We found that negative vorticity $(\Omega>0)$ reduces the wave height whereas positive vorticity $(\Omega<0)$ increases the wave height. Numerical and analytic instability growth rates of transverse perturbations have been computed and derived, respectively. We have shown that negative vorticity amplifies the rate of growth and bandwidth of transverse instability whereas positive vorticity diminishes both the rate of growth and bandwidth.

\section{Appendix. Numerical method to solve equation (3.8)}

The eigenfunctions $\hat{u}$ are computed numerically using the interpolant function (Tang (1993); Boyd (1989); Weideman \& Reddy (2000)). 

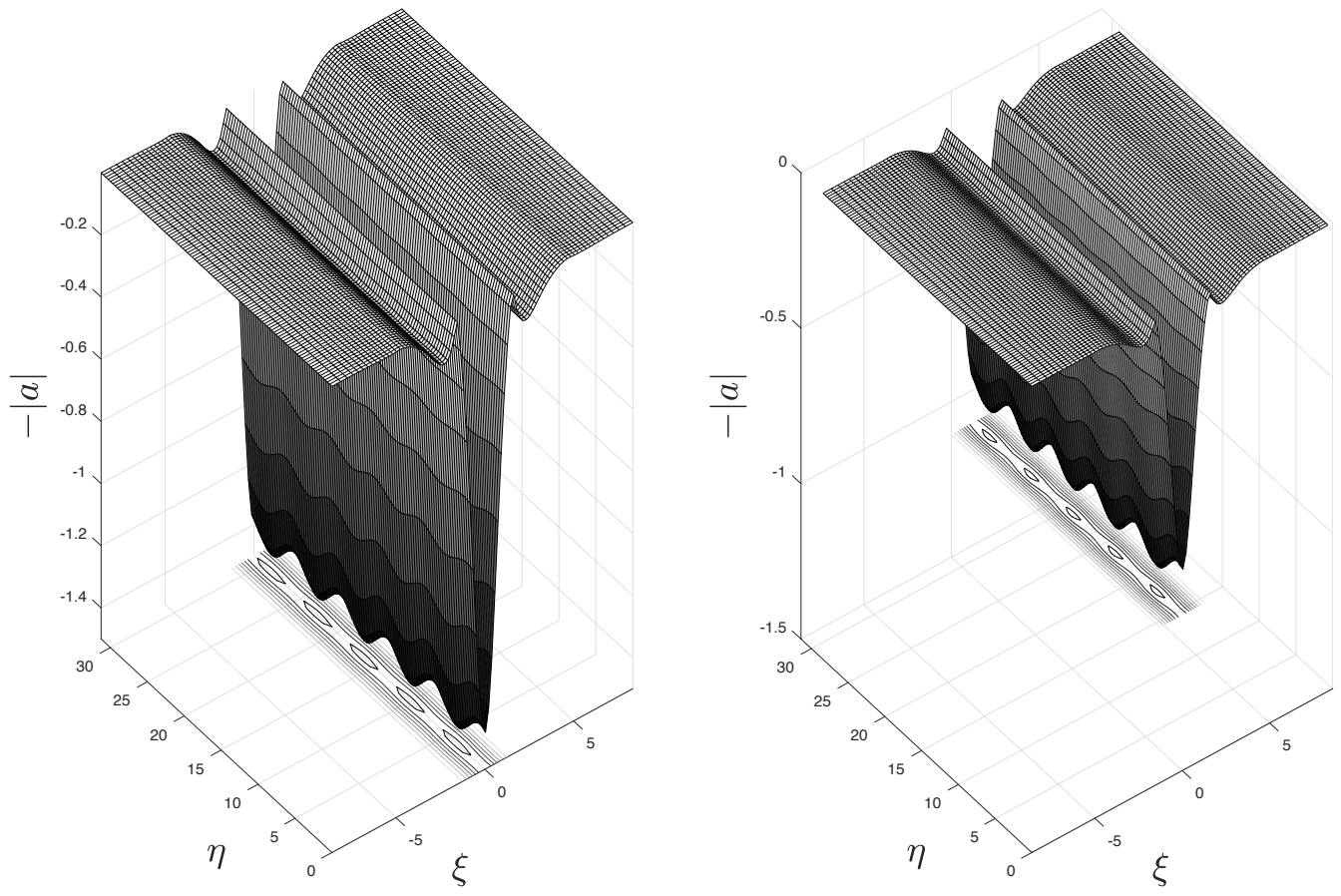

FiguRE 9. Surface elevations of perturbed envelopes of gravity-capillary solitary waves of depression in deep water for $\Omega=0, \kappa=1, q_{\max }=1.01$ (left) and $\Omega=0.10, \kappa=0.86$, $q_{\max }=1.08$ (right)

$$
\hat{u}_{N}(x)=\sum_{j=1}^{N} \frac{e^{\left(-x^{2} / 2\right)}}{e^{\left(-x_{j}^{2} / 2\right)}} \phi_{j}(x) \hat{u}_{j},
$$

where

$$
\phi_{j}(x)=\frac{H_{N}(x)}{H_{N}^{\prime}\left(x_{j}\right)\left(x-x_{j}\right)},
$$

and $H_{N}(x)$ is the Hermite polynomial of degree $N$. The grid points $x_{1}, \ldots, x_{N}$ are the roots of $H_{N}(x)$. Note that for continuous eigenfunctions on $\mathbb{R}$ vanishing at infinity they can be uniformly approximated by functions of the form $\exp \left(-x^{2}\right) p(x)$ where $p$ is a polynomial (see Lang (1991) page 62).

This spectral interpolant automatically enforces the vanishing of the eigenfunctions at $\pm \infty$. We used $N=160$ grid points giving a domain half size $L=8.61(x \in[-L, L])$. With this choice, we obtained $\left|\hat{u}_{N}( \pm L)\right|=O\left(10^{-8}\right)$.

\section{REFERENCES}

Ablowitz, J.M. \& Segur, H. 1979 On the evolution of packets of water waves. J. Fluid Mech. 92(4), 691-715.

Akers, B. \& Milewski, P.A. 2009 A model equation for wavepacket solitary waves arising from capillary-gravity flows. Stud. Appl. Math.. 122, 249-274.

Akers, B. \& Milewski, P.A. 2010 Dynamics of three-dimensional gravity-capillary solitary waves in deep water. SIAM J. Appl. Math. 70(7), 2390-2408.

Akylas, T.R. 1993 Envelope solitons with stationary crests. Phys. Fluids A 5, 789-791.

Boyd, J.P. 1989 Chebyshev and Fourier spectral methods.. Springer-Verlag, Berlin. 
Deconinck, B., Pelinovsky, D.E. \& Carter, J.D. 2006 Transverse instabilities of deep-water solitary waves. Proc. R. Soc. A 462, 2039-2061.

Dias, F., Menasce, D. \& Vanden-Broeck, J.M. 1996 Numerical study of capillary-gravity solitary waves. Eur. J. Mech., B/Fluids 15(1), 17-36.

Dorio, J.D., Cho, Y., Duncan, J.H. \& AkYlas, T.R. 2011 Resonantly forced gravitycapillary lumps on deep water. part 1. experiments. J. Fluid Mech. 672, 268-287.

Hsu, H-C., Kharif, C., Abid, M. \& Chen, Y.Y. 2018 A nonlinear schödinger equation for gravity-capillary water waves on arbitrary depth with constant vorticity: Part i. J. Fluid Mech. 854, 146-163.

Iooss, G. \& Kirchgässner, K. 1990 Bifurcation d'ondes solitaires en présence d'une faible tension superficielle. C.R. Acad. Sci. Paris, Série I 311, 265-268.

KIM, B. 2012 long-wave transverse instability of weakly nonlinear gravity-capillary solitary waves. J. Eng. Math. 74, 19-28.

Kim, B. \& Akylas, T.R. 2005 On gravity-capillary lumps. J. Fluid Mech. 540, 337-351.

Kim, B. \& Akylas, T.R. 2007 Transverse instability of gravity-capillary solitary waves. J. Eng. Math. 58, 167-175.

LANG, S. 1991 Real and functional analysis.. Springer-Verlag, New-York.

Longuet-Higgins, M.S. 1989 Capillary-gravity waves of solitary type on deep water. J. Fluid Mech. 200, 451-470.

Longuet-Higgins, M.S. 1993 Capillary-gravity waves of solitary type and envelope solitons on deep water. J. Fluid Mech. 252, 703-711.

Longuet-Higgins, M.S. \& Zhang, X. 1997 Experiments on capillary-gravity waves of solitary type on deep water. Phys. Fluids 9, 1963-1968.

MASNADI, N. \& DunCAN, J.H. $2017 a$ The generation of gravity-capillary solitary waves by a pressure source moving at a trans-critical speed. J. Fluid Mech. 810, 448-474.

Masnadi, N. \& Duncan, J.H. 2017b Observation of gravity-capillary lump interactions. J. Fluid Mech. 814, R1.

Milewski, P.A. 2005 Three-dimensional localized solitary gravity-capillary waves. Comm. Math. Sci. 3(1), 89-99.

Milewski, P.A. \& WAng, Z. 2014 Transversally periodic gravity-capillary solitary waves. Proc. R. Soc. A 470, 20130537.

Parau, E.I., Vanden-Broeck, J.M. \& Cooker, M.J. 2005 Nonlinear three-dimensional gravity-capillary solitary waves. J. Fluid Mech. 536, 99-105.

PARK, B. \& CHO, Y. 2016 Experimental observation of gravity-capillary solitary waves generated by a moving air suction. J. Fluid Mech. 808, 168-188.

RYPDAL, K. \& RASMUSSEN, J.J. 1989 Stability of solitary structuresin the nonlinear schrödinger equation. Phys. Scripta 40, 192-201.

Saffman, P.G. \& Yuen, H.C. 1978 Stability of a plane soliton to infinitesimal two-dimensional perturbations. Phys. Fluids 21, 1450-1451.

TANG, T. 1993 The hermite spectral method for gaussian-type functions. SIAM J. Sci. Comput. 14, 594-606.

Vanden-Broeck, J.M. \& Dias, F. 1992 Gravity-capillary solitary waves on water of infinite depth and related free surface flows. J. Fluid Mech. 240, 549-557.

WANG, Z. \& Milewski, P.A. 2012 Dynamics of gravity-capillary solitary waves in deep water. J. Fluid Mech. 708, 480-501.

Weideman, J.A. \& REDDy, S.C. 2000 A matlab differentiation matrix suite. ACM transactions on mathematical software(TOMS) $\mathbf{2 6 ( 4 )}$.

Zakharov, V.E. \& RubenchiK, A.M. 1974 Instability of wave guides ans solitons in nonlinear media. J.E.T.P. 38, 494.

ZhANG, X. 1995 Capillary-gravity and capillary waves generated in a wind-wave tank: observations and theories. J. Fluid Mech. 289, 51-82.

ZHANG, X. 1999 Observations on waveforms of capillary and gravity-capillary waves. Eur. J. Mech.-B/Fluids 18, 373-388.

Zhang, X. \& Cox, C.S. 1994 Measuring the two-dimensional structure of a wavy water surface optically: a surface gradient detector. Exps. Fluids 17, 225-237. 